\title{
Health Care Providers' Stigma Against HIV-positive Women: A Phenomenography Qualitative Research in Iran
}

\section{Samira Jafari ${ }^{1}$, Tahmineh FarajKhoda ${ }^{2}$, Mohammad-Hossein Baghiani Moghadam', Minoo Mohraz}

'Department of Health Education and Promotion, School of Public Health, Shahid Sadoughi University of Medical Sciences, Yazd, Iran

${ }^{2}$ Research Center for Nursing and Midwifery Care, Shahid Sadoughi University of Medical Sciences, Yazd, Iran

3ranian Research Center for HIV/AIDS, Iranian Institute for reduction of High- Risk behaviors, Tehran University of Medical Sciences, Tehran, Iran.

Study Area: Tehran, Iran

Coordinate: $29^{\circ} 29^{\prime} 47^{\prime \prime} \mathrm{N} 60^{\circ} 51^{\prime} 46^{\prime \prime E}$

Key words: AIDS, WLWH (Women Living with HIV), Medical ethics, PTSD (post-traumatic stress disorder)

\begin{abstract}
HIV/AIDS-related stigma can affect the diagnosis, treatment and the prevalence of HIV. Health providers' stigma can be fatal in this regard. In this study, we have tried to explore the perceptions of health providers' stigma against HIV/AIDS among HIV-affected women. Here we applied a phenomenography approach. Samples were selected purposively from the behavioural disorders clinic and HIV positive club of Iranian Research Centre for HIV/AIDS (HPC). In-depth interviews were conducted for at least one hour with 14 healthcare providers (HCP), 14 women living with HIV and 7 family members. Data collections and analysis were conducted at the same time using a seven-step Sjöström process. Women experienced health providers' stigma in two contexts: i- Professional Level (ethics, relations and duties); ii- Institutional Level (support, education/research, monitoring and services). Wefound lack of communication skills and professional ethics. To remove stigma, we need to evaluate, modify and design various organisational programs with its relevant applications .
\end{abstract}

likely to be adversely affected by stigma; depression and PTSD (post-traumatic stress disorder) are the most common mental disorders among infected women (Visser et al., 2009). Some disease such as STD/SDI, impose a huge social burden connected to several factors (Longo et al., 2012). Diseases which are incurable, have visible symptoms, and for which the infected individuals are marked as responsible for spreading it, are more potential for stigmatisation. AIDS fits perfectly into this category and carries the significant stigma (Brimlow et al., 2014; Littlewood et al., 2008; Vanable et al., 2006). Stigma is one of the factors that adversely influence population health (Hatzenbuehler et al., 2009). Stigma usually interferes with healthcare plans (Jafari, 2016; Mbatha, 2013; Ghabili et al., 2008) and is an inhibiting factor of its prevention program (Hatzenbuehler et al., 2009, 2013; June 1999). HIV/AIDS-related stigma would result in refusal of diagnostic testing, hiding HIV status from sex partners or non-compliance to treatment, which would lead to increased prevalence of HIV/AIDS (Longo et al., 2012; Parker \& Aggleton, 2003 ). HIV/AIDS is so highly stigmatised that the effects are not limited to those who

*Corresponding Author: baghianimoghadam@yahoo.com 
are infected but it further influences people who are related to them such as caregivers, professionals (Jafari, 2017) and respective families (Jafari, 2016; Herek, 1997). Studies have revealed that even people referring to AIDS prevention clinics are stigmatized; the society and family treats them as an infected person (Nyblade et al., 2011). Thus by the social behavious and judgement, caregiver's attitude toward HIV get often negative (Steward et al., 2008). This may express itself in HIV-related stigma among HIV Care program (HCP) (Jafari et al., 2016). Three major sources of stigma in healthcare centres are- the lack of stigma knowledge, a fear of Infection, and the labelling HIV infected as immoral. It seems that stigma is mostly associated with attitude rather than knowledge and fact (Waluyo et al., 2014). Nyblade et al. (2011)'s research shows that it has a major role in volunteers' fear of potential disclosure of HIV/AIDS status and hence unwillingness to take tests. We have observed a similar situation in Iran. Consequently, potential cases are not diagnosed during the most critical stage (Valdiserri, 2002). It has been realized that theyounger individuals are deeply concerned about health providers' attitude and are less likely to refer to centres where they expect to be judged by sexual behaviours or drug use (June, 1999). The HIV treatment process would not be started before diagnosis (Valdiserri, 2002) and late diagnosis comes with less effectiveness and more cost. The efficiency of health-care services depends partly on health providers. Their stigmatised attitude or inappropriate behaviour in the workplace can create stigmatised beliefs in public (HCP's role as key informants). There are few studies which addressed the stigma experiences while interactions with health-care providers. The present piece of work tries to critically evaluate HCPs' stigma against HIV-infected women or Women Living with HIV (WLWH). We tried to investigate the stigma experience in WLWH.

Evaluating the factors associated with stigma among caregivers of HIV-positive women can help to plan antistigma programs and propose interventions aimed to mediate the attitude towards WLWH in healthcare environments.

\section{Methodology:}

Design: it was a qualitative study with phenomenography approach. Data collection was conducted in the year 2015 from the Behavioural disorders clinic and HIV Positive Club (HPC) of Iranian Research Centre including ImamKhomeini Hospital, Tehran, the capital city of Iran for HIV/AIDS. Phenomenography qualitatively investigates the different ways in which people experience phenomena to develop a clear image of the phenomena (Marton, 1994). A concept and the aim is to explain it from the perspective of the people involved (not from an expert's perspective; Marton, 1981). The experiences of the people involved are collected and linked with an outcome space (big-picture framework; Marton, 1994).

Participant enrolment: three groups were examined; HIV-infected women, the status of their family members, and HCP (HIV Care Program).Total 14 infected females, 7 family members and $14 \mathrm{HCP}$ were included in the study. Spouse, children, mother, sister, brother, and sister-in-low were also included as family members. HCP included faculty members, gynaecologist, dentists, nurses, key informants (medical infection specialist, HPC, head, and consultant, psychologist, head of the communicable disease control unit at the ministry of health), medicine experts, welfare trainer, and lab sampler. In this article we used the terminology 'Health Care Providers' instead of Health care staff or personnel.

Data collection: collected data were based on a purposive sampling method, 50 in-depth interviews lasted at least for an hour and were continued until saturation occurred.

Data analysis: data was performed manually using the phenomenography process at the same time as the data collection. The analysis included seven steps (Dahlgren \& Fallsberg, 1991). Validity and Reliability of Data: trustworthiness of data was evaluated according to Lincoln \& Guba (1985)'s evaluative criteria which include dependability, credibility, confirmability, and transferability (Polit \& Beck, 2006).

Ethical Consideration: all the participants were assured that the interviews were optional, conf idential, recorded conditional on their permission and would not affect the course of treatment. Name and address were not included. The independent ethical committee of Shahid Sadoughi University of Medical Sciences and Health Services approved the study procedure.

\section{Results:}

Our survey records: the age range of WLWH was 27-56 with an education from primary to Bachelor's degree. Stigma-related concepts were divided into 2 categories: i. professional; ii. institutional. A total of 8 categories were created; 3 categories in professional level (tableı) and 4 categories in institutional level (tablez).

\section{i. Professional Level:}

Table-1: HCP' stigma against WLWH in Professional Level

\begin{tabular}{ll}
\hline Main Category & Subcategories \\
\hline Professional & $\begin{array}{l}\text { Lackof Confidentiality } \\
\text { Ethics }\end{array}$ \\
& Make them Feel Indebted \\
& Unconventional Requests of Employees \\
\hline Professional & Improper Interaction \\
Relations & Pervasive Fear of Transmission and \\
& Infected Individuals \\
& Unpleasant Feelings \\
\hline Professional & Medical Negligence \\
Duties & Consultant Negligence \\
& Clinical Negligence
\end{tabular}




\section{Lack of Education}

The most frequent concepts of the professional level were judgmental behaviour, pervasive fear of infection and negligence in treatment.

\section{a. Professional ethics:}

1-Lack of Confidentiality: in some inpatient wards, experiences of disclosure to family members or friends are reported. A consultant stated- "In a women's ward, patient Communication Boards clearly stated HIV-infected".

2-Make them Feel Indebted: according to interviews from both staff and WLWH, providing health services are considered a favour or a form of the dedication of health providers. A lab sampler said- "We are sacrificing our lives here, the sampling unit should have been shut down".

3-Unconventional Requests of Employees: care providers demand special job conditions and organizational advantages over other colleagues for their care and treatment of HIV/AIDS patients. To quote one of the lab samplers-"We should be financially supported or have some behavioural support. We should have full authority at this centre and no one should order us...". At some private clinics (e.g. dental clinics), clients are asked to pay a higher price than routine due to HIV condition.

4- Renege (putting off): doctors and dentists are bound to treat them, then kept them waiting, and often did not accept them. A woman had most her teeth broken off, leaving only the root behind, she opened her mouth and said, "Just like a ball, they throw us up and down, they play with us, a dentist told me to sit down and wait. I waited until $10 \mathrm{pm}$ then he told me he would not pull my teeth because I have HIV.... In a participant observation, we (author) introduced ourself as an HIV-infected and none of the dentists accepted her.

5-Judgment: “We tend to generalise an individual's mistake to a population. Unfortunately, in this context, we, HCP, think that the disease is a result of that mistake", said a psychologist of HPC; Evidence strongly suggests that HCP's attitude towards WLWH is very judgmental. "Diabetes occurs unintentionally but it is conscious and immoral" (expert). While speaking with the researcher, the head of nursing school pointed out that HIV+ individuals seek revenge through infecting others, "Wow... you are so brave. You must be very careful... they generally seek revenge". "I blame them a lot; they are guilty because they are going to infect others" (A nurse); Several respondents mentioned that such groups $(\mathrm{HIV}+)$ are regarded sinful people who either have multiple extramarital sex partners or are sexworkers.

\section{b- Professional Relations:}

1-Improper interaction: improper interactions included unkindness, lack of empathy, and insult, discrimination, curiosity, avoidance, disrespect, sense of insecurity, and labeling (using the word AIDS instead of Patient). Body language showing humiliation such as shouting, frowning, whispering etc. "Why aren't we kind to them ... we can make HIV/AIDS persons happy, when they are happy, they will forget about HIV and AIDS". (Domestic services staff). An ICU nurse stated that- "These people should be denied... we should discriminate and separate them like leprosy patients ...those who are sinned should be labelled as secondary".

Not only patients, but also HIV-positive employees of the centre should be discriminated. "When there is a birthday party, they won't invite my colleague $(H I V+)$, but they would invite other (not $H I V+)$ ". "Out of curiosity, they (HCP) may go through her background information to find out how she is infected". (Nurse)

2-Pervasive Fear of transmission and infected individuals: in this study, almost all HCP, including physicians, surgeons, head and members of the nursing faculty, nurses and even domestic services staff were deeply worried about infection. Fear of HIV transmission was more than Hepatitis transmission. A lab sampler has recently experienced needle-stick injury and has taken preventive medications. She said- "I am still much stressed, what if the medication doesn't work... it has totally messed my mind up." Stigma is not restricted to fear of transmission. Respondents reported fear of infected individuals, and viewed them as harmful and dangerous- "It seems to you as if they are always about to spit. I am always stressed".

3-Unpleasant feelings: Women perceived feelings of anger and hatred from HCP. "I am just doing my job; there is no feeling of love. I don't like them;... for other markers you don't feel like you hate the person" (Staff).

\section{c- Professional Duties:}

1-Medical Negligence: WLWH were frustrated by the rejection of doctors and dentists. "I went to dentistry once, he threw me out of his office with pence" (28year-old woman).

2- Consultant negligence: the majority of women felt the need to consult with a health advisor (consultant). But the required consulting service was either not provided or not continued. There was even no consoling after HIV-test notification of a positive test result. "...he has been infected for 5 years and no consoling yet" (Consultant).

3- Clinical negligence: clinical negligence was frequently mentioned. Here we are mentioning some quotations regarding delay and negligence in care services for inpatients ward. In some hospital wards, critically ill patients are not admitted. For example, a nurse at infectious disease ward explained, "These patients are hardly ever accepted to other wards, I had an HIV+ patient who stayed here for 2-3 months, and was intubated here...."

4-Lack of education: WLWH and their respective family members have the right to be knowledgeable, but they are not receiving proper education about infection, disease, prevention of transmission, treatment, 
adaptation, the possibility of having children, etc. None educated patient stated- "this is the fourth time I am stopping any medicine... I think they are shortening my Life" (Sara-32years).

Lack of information and misleading information cause strict behaviour or wrong reactions among families and have created uncomfortable and humiliating feelings for women.

\section{ii. Institutional Level:}

Tablez- HCP' stigma against WLWH in Institutional Level

\begin{tabular}{|c|c|}
\hline Main Category & Subcategories \\
\hline Support & $\begin{array}{l}\text { Concealing HIVStatus } \\
\text { Reluctance to Join Community-based } \\
\text { Activities } \\
\text { Lack of NGO*s Support } \\
\text { Lack of Financial Support }\end{array}$ \\
\hline $\begin{array}{l}\text { Education and } \\
\text { Research }\end{array}$ & $\begin{array}{l}\text { Academic Education } \\
\text { Continuing medical education } \\
\text { Lack of public education } \\
\text { Research Limitations }\end{array}$ \\
\hline Monitoring & $\begin{array}{l}\text { Monitoring Code of Ethics and } \\
\text { Professional Conduct } \\
\text { Monitoring Treatment } \\
\text { Addressing Violations and } \\
\text { Resolving Complaints infected individuals }\end{array}$ \\
\hline Services & $\begin{array}{l}\text { Lack of available centers and workforce } \\
\text { Inadequate Equipmentat Centers \& Club } \\
\text { Deficiencies in Provision of Dental and } \\
\text { Consulting Services }\end{array}$ \\
\hline
\end{tabular}

\section{a-Support}

*Non-Government Organization

1- Concealing HIV status: HIV/AIDS infection is not considered a crisis yet and is not receiving the deserved attention. The true number of infected individuals in the country is uncertain. As per HCP, "International AIDS day was removed from our calendars". The majority of women in our study felt neglected and dissatisfied. "We are humans too... come give us a visit too... you go abroad much often, though".

2- Reluctance to join community-based activities "They don't let my colleagues to teach" (Consultant). Despite their efforts, HPC members are not permitted to provide educational services for public, schools, universitystudents, etc.

3- Lack of NGOs support: there are few NGOs in the country supporting infected individuals, most of them are not dedicated to women and involves both men and women. Governmental support centres are also limited.

4- Lack of Financial Support: there are no financial aid programs to support HIV-infected women or create jobs for them.

\section{b- Education and Research}

1- Academic education: an infected woman addressed the educators- "you should start with yourself, remove the stigma and discrimination from universities, teaching should start at academic level...learn good conduct and proper behaviour. Our infection should not be a reason to avoid us". Educators teach with stigmatised attitude. Profession ethics, stigmatised diseases, and anti-stigma programs are not aimed syllabus of the medical education system.

2- Continuing medical education: "... We have no protocols but the infection control supervisor teaches us what to do after a needle-stick" (Hospital supervisor). In health centres, there were no programs or protocols in terms of destigmatizing, life with HIV, updated information about care and treatment, prevention of transmission. A mother believes the physicians did not have the necessary information to prescribe medication for her HIV+ daughter.

3- Lack of public education: "Once a year we send an SMS to all cell numbers". A quote from an off icial, declaring that public education is limited to that one day.

4- Research Limitations: a researcher should confront several obstacles to conduct a research on HIV/AIDS.

\section{c-Monitoring}

1- Monitoring code of ethics and professional conduct: no protocols have been developed to monitor the professional conduct of HCP.

2- Monitoring treatment: this is how a client describes the process to get medication from the centre- "The previous expert would ask us some questions and write something in our booklet....".

$3^{-}$Addressing violations and resolving complaints: infected individuals are no aware of their rights to receive health-care services and treatment.

\section{d-Services}

1- Lack of available centres and workforce: for most regions, there was only one behavioural disorders clinic in the capital of the province. WLWH need to travel long distances to receive services.

2- Inadequate equipment at centers and clubs: according to the clients, club location is physically unfit and amenities and extra-curricular programs are basic.

3- Deficiencies in provision of dental and consulting services: most provinces did not offer dental and consulting services.

\section{Discussion:}

\section{i. ProfessionalLevel}

At the professional level, ethics, relations, and duties of HCP were overshadowed by stigma. 


\section{a- Professional Ethics:}

Nondisclosure, make them feeling indebted, renege, unconventional requests, and judgment on employee's side.

Lack of confidentiality: as per (Abbasi et al., 2008) disclosure of personal secrets is a barrier to prevention and care. Revealing patient's HIV infection (to a family member, relatives and even HCP) at the hospital usually distorted women's sense of security and conf idence. Make them feel indebted and unconventional requests: fear and notions of suicide caused due to such unconventional requests by $\mathrm{HCP}$ at governmental and private sectors.

Renege: despite the promise to help women renege on treatment; They perceived being re-assigned to different employees (Rahmati et al., 2012) so they felt hopeless (Karamouzian et al., 2015) rejected, angry, and discriminated (Jafari, 2016, Rahmati et al., 2012).

Judgment: judgment is recognised as a barrier to prevention and care (Abbasi et al., 2008). Judgment has challenged the judgment-free treatment. The most common manifestation of stigma not only in society but also among HCP is judgment (Jafari et al., 2016). Judging has overshadowed the thoughts, attitudes and perception of truth, morality and personal behaviour (Steward et al., 2008); in so far as that the HCP believed in maintaining the stigma, isolating them from society and even wished them death (Jafari, 2016, Rahmati et al., 2012). In this study, the concept of conviction is regarded as a highlight of judgmental beliefs (Rahmati et al., 2012). According to our experience, a conviction for HIV-infection was stronger that conviction for real criminals (prisoners who are transferred to the centre).

\section{b-Professional Relations}

Improper interactions and unpleasant feelings: sometimes HCP shows discriminatory attitudes towards HIV-infected (Karamouzian et al., 2015, Rahmati et al., 2012, Waluyo et al., 2014, 2010, Bukar et al., 2008, Askarian et al., 2006, Vanable et al., 2006). The stigmatised behaviour of HCP as experts made the WLWH realise the seriousness of the situation and induce hopelessness (Rahmati et al., 2012). Such that, mothers experienced hesitation and failure in neonatal care- "Bury your child alive, go bury it...". HCP encouraging to abortion and sterilisation. In accord with other studies, we believe that fear of discrimination is a determinant factor in a disclosure of HIV or pretending to have Hepatitis B and D (Rahmati et al., 2012).

Transmission obsession (fear): there was an Extreme fear of infection, (Karamouzian et al., 2015, Rahmati et al., 2012) which the authors call "transmission obsession", that affect their behaviour and care quality. The fear resulted in avoidance reactions. To this end, the most comment prevention method among health professionals are staying away and avoiding physical contact (Rahmati et al., 2012). Low HIV/AIDS knowledge among HCP is a possible reason (Balfour et al., 2010, Askarian et al., 2006). Although in our study HCP reaction did not depend on education level, position and job rank, it is noteworthy and should be studied in more details.

\section{c-Professional Duties}

Medical negligence: similar to reports from other countries, an outstanding finding of the current work was a HIV-related stigma among dentists (Karamouzian et al., 2015, Zukoski \& Thorburn, 2009, Bukar et al., 2008). Ostracism is a complex issue, where professional ethics and duties both are challenged. Refusing to treat WLWH questions on ethical issues where sterilisation factors are a part of their duties. Breakdown of instruments (due to the frequent use of an autoclave) and the cost of replacing them urge the dentists to refuse treatment. Thus they choose the easiest solution; rejection. In a dentist's perspective, rejection is a responsible act not a violation of ethics.

\section{ii. Institutional Level}

\section{a-Support}

It seems that Lack of Financial Support, Reluctance to positive people,s Community-Based Activities and Lack of NGO,s Support were consequences of conealing.

\section{b- Education and research}

Education influences and is influenced by stigma at every level (academic, re-training HCP and public).

Academic education: medical education curriculum is unsuccessful in knowledge transfer from evidence to practice (Karamouzian et al., 2015). This gap between theory and practice along with a lack of knowledge are the reasons for stigmatised behaviour (Karamouzian et al., 2015). Our scholars and researchers consider the HIVinfected as Patients. Even in some international papers, HIV-infected are mentioned as Patients. There seems to be an organisational culture in this context. We may speculate that the importance of learning professional ethics is not appreciated. Ethical educations should not be restricted to theory and teachers and HCP's behaviour should exhibitanideal.

Continuing medical education: currently, re-training programs to stay updated with health care changes and reduce stigma among $\mathrm{HCP}$ are not a priority. This is associated to a series of problems for HIV-positive individuals. Continuing medical education has seen effective in stigma management (Karamouzian et al., 2015).

Lack of Public Education: health system is dealing with restrictions in community-based prevention programs. Public HIV education through mass media may be 
regardedas promoting such behaviors.

Research: individual and institutional stigma is the main reason why there have been few studies on this topic (Jafari, 2016). Researchers are not very fond of the topic because of organisational barriers such as strict licensing and individual stigmas, such as unpleasant feelings, fear of infected individuals and HIV transmission, and unwillingness to contact with PLWH.

\section{c-Monitoring:}

It is not possible to file/address a complaint against improper interactions, unconventional fees, and the refusal of treatment. Furthermore, PLWH is not aware of their rights. These work hand in hand to provoke stigmatising reactions.

\section{d-Services:}

Involving women in planning, conduct and monitoring of HIV-related programs (Abbasi et al., 2008) and Infrastructure requirements are also necessary. For instance, privacy is while visiting the WLWH is an important factor. An infected woman who visited a paediatric stated that "... I did not tell him...(when there were other patients in the room)...I would have felt uncomfortable."

Lack of consulting services and workforce: receiving and providing services may be delayed, because of a limited number of counselling centres. Abbasi et al. (2008) suggested that lack of access to health services and information would increase the vulnerability of women and girls to HIV-related. Heavy workload along with low workforce has created complications. As an example, a centre did not provide counselling service because the consultant was busy, filling another position with higher priority.

Study limitations: despite the wide diversity of participants at a Referral Centre of the country, participation of other ethnic groups of Iran such as Baluch could have created a broader and deeper notion of phenomena.

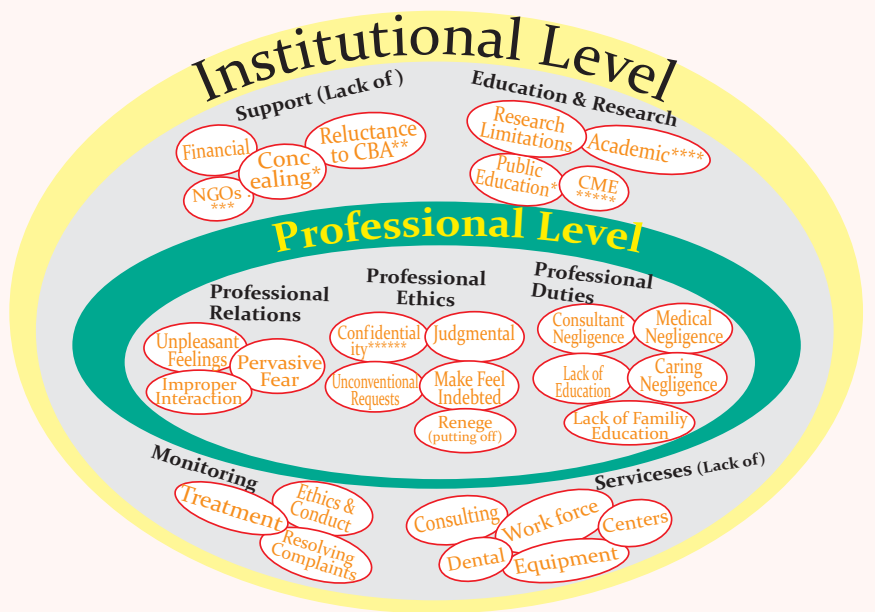

Figure-1: Overall situations which the HIV/AIDS affected patients are facing

Legends:

*Concealing HIVStatus

** Reluctance to Join Community-based Activities

$* * *$ Lack of NGOs Support

$* * * *$ Academic Education

$* * * * *$ Continuing Medical Education

$* * * * * *$ Lack of Conf identiality

\section{Conclusion:}

Till date, the present taken issue has received very less attention. Removal of international AIDS Day from country's official calendar, lack of a well-established national HIV/AIDS treatment (in private clinics) and care protocol, lack of anti-stigma training for HCP are overall indicating a highly stigmatised system. This phenomenon is known to be a lifestyle disease and a result of their conscious choice. Therefore, a person affected with HIV is blamed and condemned to disrespect, hatred and discrimination.This phenomenon has turned into the disease of terror and secret; HIV infection is deemed a crime, an HIV-positive individual is considered a criminal, HCP fear transmission and WLWH fear disclosure. The backbone of stigma was a judgment; it had challenged professional ethics and influenced professional relations through (unpleasant) feelings (Fig.-1).

\section{Recommendations:}

We need to pay more attention to medical education, retraining of HCP, public education. In ethics education for students, teachers are to be considered as a model for their student. Development and implementation of treatment and professional conduct monitoring programs (with enforcement) can effectively modulate stigma and its consequences.

\section{Acknowledgements:}

The authors would like to thank all participants for their kind collaboration. We would also like to show our gratitude to professor Dr Ehrampoush, for his assistance and moral support.

\section{References:}

Abbasi, M., Abbasian, L.,Ehsanpour, S.R. \& Ghanbari, A. (2008): HIV Transmission by Sexual Intercourse and Blood Transfusion Regarding to Civil Effects and Criminal Law. Iran. J. Med. Law, 2:129-167.

Askarian, M., Hashemi, Z., Jaafari, P. \& Assadian, O. (2006): Knowledge about HIV Infection and Attitude of Nursing Staff Toward Patients with AIDS in Iran. Infect. Control Hosp. Epidemiol. 27(1):48-53

Balfour, L., Corace, K., Tasca, G.A., Best-Plummer, W., Macpherson, P.A. \& Cameron, D.W. 2010. High HIV knowledge relates to low stigma in pharmacists and university health science students in Guyana, South America. Int. J. Infec. Dis., 14, e881-e887.

Brimlow, D., Cook, J. \& Seaton, R. (eds.) (2014) Stigma and 
HIV/AIDS: a review of the literature 2oo3: Rockville, MD: US Department of Health and Human Services.

Bukar, A., Gofwen, R., Adeleke, O.A., Taiwo, O.O., Danfillo, I.S. \& Jalo, P.H. (2008): Discriminatory attitudes towards patients with HIV/AIDS by dental professionals in Nigeria. Odontostomatol. Trop., 31(122):34-40.

Dahlgren, L. O. , \& Fallsberg, M. (1991): Phenomenography as a qualitative approach in social pharmacy research. J. Soc. Admin.Phar., 8(4), 150-156.

Ghabili, K., Shoja, M. \& Kamran, P. (2008): The Iranian female high school students' attitude towards people with HIV/AIDS: a cross-sectional study. AIDS Res. Ther., 5 (15).

Gross, J.J. \& Muñoz, R.F. (1995): Emotion regulation and mental health. Clin. Psychol-Sci.Pr., 2(2):151-164.

Hatzenbuehler, M.L., Nolen-Hoeksema, S. \& Dovidio, J. (2009): How does stigma "get under the skin"?: the mediating role of emotion regulation. Psychol. Sci., 20(10):1282-1289.

Hatzenbuehler, M.L., Phelan, J.C. \& Link, B.G. (2013): Stigma as a Fundamental Cause of Population Health Inequalities. $\underline{A m}$. J. Pub.Health, 103:813-821.

Herek, G.M. (1997): Stigma and sexual orientation: Understanding prejudice against lesbians, gay men and bisexuals 4. Pub.: SAGE Publications, Inc.,

Jafari, S. (2016): Developing Stigma Scale for HIV/AIDS Based on Phenomenographic Study, Psychometry and Assessment of it,s Prevalance in Affected Women Reffered to Iranian Research Center for HIV/AIDS and Yazd Consulting Center for Behavioral Disorders,2014 to 2016. Ph.D, thesis, Shahid Sadoughi University of Medical Sciences and Health Services.

Jafari, S., Farajkhoda, T., Baghianimoghadam, M-H. \& Mohraz, M. (2016): HIV positive women's Experiences of Stigma: health care provider's stigmatized professional relationship. The fourth annual congress of mediacal ethics and fourth congress of nursing ethics. Tehran, Iran: Journal of Medical ethics and history of medicine.

Jafari, S. (2017). HIV Stigma Prevalence in Yazd Province. International Congress on Addiction And High Risk Behaviour, 3-4 May, 2017, Yazd, Iran

Karamouzian, M., Akbari, M., Haghdoost, A.A., Setayesh, H. \& Zolala, F. (2015): I Am Dead to Them: HIV-related Stigma Experienced by People Living With HIV in Kerman. Iran. I Assoc. Nurses AIDS Care, 26(1):46-56.

Lincoln, Y.S., \& Guba, E.G. (1985). Naturalistic inquiry. Beverly Hills, CA:Sage.

Littlewood, R.A., Vanable, P.F., Carey, M.P., Blair, D.C. \& Blair, D.C. (2008): The association of benef it finding to psychosocial and health behavior adaptation among HIV+ men and women. LBehav Med., 31(2):145-55.
Longo, D. L., Kasper, D. L., Jameson, J. L., Fauci, A. S., Hauser, L. S. \& Loscalzo, J. (2012): Harison,s Principles of Internal Medicine, McGraw-Hill Professional.

Marton, F. (1981): Phenomenography describing conceptions of the world around us. Instr. Sci., 10(2):177-200.

Marton, F. (ed.) (1994): Phenomenography.In, in Huse'n T. \& Postlethwaite T.N. (eds): The International Encyclopedia of Education. Pub.: Pergamon Press, Oxford.

Mbatha, B. (2013): AIDS-Related Stigma as a Barrier to HIV and AIDS Prevention, Care and Treatment in South African Public Universities. Mediterr. J. Soc. Sci., 4(14):517-524.

Nyblade, L., Singh, S., Ashburn, K., Brady, L. \& Olenja, J. (2011): "Once I begin to participate, people will run away from me": Understanding stigma as a barrier to HIV vaccine research participation in Kenya. Vaccine, 29(48):8924-8928.

Parker, R. \& Aggleton, P. (2003): HIV and AIDS-related stigma and discrimination: a conceptual framework and implications for action. Soc. Sci. Med., 57(1), 13-24.

Polit-O'Hara, D. \& Beck, C. (2006): Essentials of nursing research methods, appraisal, and utilization. Vol.:1. Pub.: Philadelphia, Lippincott Williams \& Wilkins.

Rahmati, F., Niknami, S., Amin, F. \& Ravari, A. (2012): HIV/AIDS Patients' Experiences about Stigma: a Qualitative Study. I. Qual. Res. Health Sci., 1(2):71-80.

Skinner, D. \& Mfecane, S. (2004) Stigma, discrimination and the implications for people living with HIV/AIDS in South Africa. Sahara J. 1(3):157-164.

Steward, W., Herek, G., Ramakrishna, J., Bharat, S., Chandy, S. \& Wrubel, J. (2008): HIV-related stigma: adapting a theoretical framework for use in India. Soc. Sci. Med., 67(8):1225-1235.

Valdiserri, R.O. (2002): HIV/AIDS Stigma: An Impediment to Public Health. Am. J. Pub. Health, 92(3):341-342.

Vanable, P.A., Carey, M.P., Blair, D.C. \& Littlewood, R.A. (2006): Impact of HIV-related stigma on health behaviors and psychological adjustment among HIV-positive men and women. AIDSBehav., 10(5):473-482.

Visser, M.J., Makin, J.D., Vandormael, A., Sikkema, K.J. \& Forsyth, B.W. (2009): HIV/AIDS stigma in a South African community. AIDS Care, 21(2):197-206.

Waluyo, A., Culbert, G., Levy, J. \& Norr, K. (2014): Understanding HIV-related Stigma Among Indonesian Nurses. I. Assoc. Nurses AIDS Care, 26(1):69-80.

Zukoski, A.P. \& Thorburn, S. (2009): Experiences of stigma and discrimination among adults living with HIV in a low HIVprevalence context: A qualitative analysis. AIDS Patient CareSTDS. 23(4):267-276. 